\title{
The Averaged Lagrangian and High-Frequency Gravitational Waves *
}

\author{
M. A. H. MacCallum ${ }^{\star}$ and A. H. Taub \\ Department of Mathematics, University of California, Berkeley
}

Received October 27, 1972

\begin{abstract}
The averaged Lagrangian technique of Whitham is applied to the second variation Lagrangian for the perturbations of a general-relativistic spacetime. This gives a variational principle for (sums of) approximately periodic gravitational waves which in turn leads to the rederivation of some results of Isaacson. Examples of the use of the method are discussed.
\end{abstract}

\section{Introduction: The Averaged Lagrangian Technique}

The purpose of this paper is to discuss approximate solutions of the Einstein equations which are approximately periodic (and can be interpreted as containing high-frequency gravitational waves) by the "averaged Lagrangian" method introduced by Whitham [1]. Whitham showed that this method is closely related to the so-called "two-timing" method, which has been used for the gravity-wave problem by ChoquetBruhat [2] and Madore [3]. In this introduction we will review these techniques, in Section 2 we discuss gravitational waves and the Lagrangian for them, and in Section 3 we apply the averaging technique to this Lagrangian. Some examples are discussed in Section 4.

The two-timing method consists of assuming that changes in the dependent variables, $\psi^{A}$ say, of a problem occur on two scales; for example, a wave train may show rapid oscillation and a slow change in amplitude, frequency and wave number. One writes

$$
\psi^{A}=\psi^{A}\left(X^{v}, \theta\right)
$$

where $X^{\mu}=\varepsilon x^{\mu}$ and $\theta=\varepsilon^{-1} \Theta\left(X^{\mu}\right)$ and it is assumed that the derivatives of $\psi^{A}$ with respect to $X^{\mu}$ and $\theta$ are of equal magnitude (which we may take as order unity). The small parameter $\varepsilon$ then measures the ratio of the fast length scale to the slow one. It should be noted that rapid variations only occur in the direction of the vector

$$
l_{\mu}=\frac{\partial \theta}{\partial x^{\mu}}=\frac{\partial \Theta}{\partial X^{\mu}}=\Theta_{, \mu} .
$$

* Supported by National Science Foundation grant GP-31358.

$\star \star$ Permanent address: King's College, Cambridge CB 2 1 ST, U.K. 
We introduce the notation that if $f=f\left(X^{\mu}, \theta\right)$

so that

$$
\left.\begin{array}{rl}
f_{, \mu} & :=\frac{\partial f}{\partial X^{\mu}} \\
\dot{f}: & =\frac{\partial f}{\partial \theta}
\end{array}\right\}
$$

$$
\frac{\partial \psi^{A}}{\partial x^{\varrho}}=\varepsilon \psi_{, \varrho}^{A}+l_{\varrho} \dot{\psi}^{A} \text {. }
$$

We can now treat the problem as if there were five independent variables $\left\{X^{\mu}, \theta\right\}$. On making the substitution $\theta=\varepsilon^{-1} \Theta\left(X^{o}\right)$ any solution to this problem will give a solution to the original problem. The usual procedure in the two-timing method is to assume that the solution has the form

$$
\psi^{A}\left(X^{\mu}, \theta\right)=\sum_{0}^{\infty} \varepsilon^{n} \psi_{(n)}^{A}\left(X^{\mu}, \theta\right)
$$

substitute this in the governing equations, and equate to zero the coefficients of the successive powers of $\varepsilon$. One normally then has to impose further restrictions on the $\psi_{(n)}^{A}$ in order that the $\psi_{(n+1)}^{A}$ remain bounded (i.e. so that each term has its assumed magnitude), cf. [2].

Suppose that the field equations satisfied by the $\psi^{A}$ are derived from a variational principle

that is, are of the form

$$
I_{0}=\int \mathscr{L}\left(\psi^{A}, \frac{\partial \psi^{A}}{\partial x^{\varrho}}\right) d^{4} x
$$

where

$$
\frac{\partial}{\partial x^{\sigma}}\left(\pi_{A}^{\sigma}\right)-\frac{\partial \mathscr{L}}{\partial \psi^{A}}=0
$$

$$
\pi_{A}^{\sigma}=\frac{\partial \mathscr{L}}{\partial\left(\frac{\partial \psi^{A}}{\partial x^{\sigma}}\right)} .
$$

In terms of the two-timing method, (1.6) can be written

where

$$
\varepsilon \pi_{A, \sigma}^{\sigma}+l_{\sigma} \dot{\pi}_{A}^{\sigma}-\frac{\partial \mathscr{L}}{\partial \psi^{A}}=0
$$

$$
\pi_{A}^{\sigma}=\pi_{A}^{\sigma}\left(\psi^{A}, l_{\varrho} \dot{\psi}^{A}+\varepsilon \psi^{A}{ }_{,}\right) .
$$

The lowest order term in an expansion of the form (1.5) satisfies

where

$$
l_{\sigma} \dot{\pi}_{(0) A}^{\sigma}-\frac{\partial \mathscr{L}_{(0)}}{\partial \psi_{(0)}^{A}}=0
$$

$$
\pi_{(0) A}^{\sigma}=\pi_{A}^{\sigma}\left(\psi_{(0)}^{A}, l_{\varrho} \dot{\psi}_{(0)}^{A}\right) \quad \text { and } \quad \mathscr{L}_{(0)}=\mathscr{L}\left(\psi_{(0)}^{A}, l_{\varrho} \dot{\psi}_{(0)}^{A}\right) \text {. }
$$


Equation (1.9) has the first integral

$$
l_{\sigma} \pi_{(0) A}^{\sigma} \dot{\psi}_{(0)}^{A}-\mathscr{L}_{(0)}=A\left(X^{\mu}\right)
$$

where $A\left(X^{\mu}\right)$ is an arbitrary function of the variables $X^{\mu}$. The requirement that the $\psi_{(1)}^{A}$ be acceptable imposes conditions on $l_{\sigma}\left(X^{\mu}\right)$ and $A\left(X^{\mu}\right)$ in general.

Whitham [1] made the important observation that (1.7) are the Euler equations of the five-dimensional variational principle given by

$$
I=\int \mathscr{L}\left(\psi^{A}, l_{\varrho} \dot{\psi}^{A}+\varepsilon \psi^{A},{ }_{\varrho}\right) d^{4} x d \theta .
$$

In the case where the functions $\psi^{A}\left(X^{\mu}, \theta\right)$ are periodic in $\theta$, one may define

$$
\bar{L}=\frac{1}{2 \pi} \int_{0}^{2 \pi} \mathscr{L}\left(\psi^{A}, l_{\varrho} \dot{\psi}^{A}+\varepsilon \psi^{A}, \varrho\right) d \theta
$$

so that to lowest order in $\varepsilon$

$$
I=\int \bar{L}_{0}\left(l_{\sigma}, A\right) d^{4} x
$$

where $\bar{L}_{0}$ arises from the $\mathscr{L}_{0}$ of Eq. (1.10) by

$$
\bar{L}_{0}\left(l_{\sigma}, A\right)=\frac{1}{2 \pi} \int_{0}^{2 \pi} l_{\sigma} \pi_{(0) A}^{\sigma} \dot{\psi}_{(0)}^{A} d \theta-A\left(X^{\mu}\right)=\frac{l_{\sigma}}{2 \pi} \oint \pi_{(0) A}^{\sigma} d \psi_{(0)}^{A}-A\left(X^{\mu}\right)
$$

In the case where the lowest order solution of the two-timing method is periodic in $\theta$, varying $\theta$, and hence $l_{\sigma}$, and $A$ in (1.13) gives rise to the same equations for $l_{\sigma}$ and $A$ as we derive from the boundedness requirements on $\psi_{(1)}^{A}$. This will follow if, to lowest order in $\varepsilon$, the four-dimensional variational principle given by the integral $I_{0}$ is the same as that obtained from (1.11) by averaging. But Bretherton [4] proved this by showing that for any function $F\left(X^{\mu}, \theta\right)$ periodic in $\theta$, if

then, for sufficiently small $\varepsilon$,

$$
\bar{F}\left(X^{\mu}\right)=\frac{1}{2 \pi} \int_{0}^{2 \pi} F\left(X^{\mu}, \theta\right) d \theta
$$

$$
\int_{-\infty}^{\infty} F\left(X^{\mu}, \varepsilon^{-1} \Theta\left(X^{\mu}\right)\right) d^{4} X=\int_{-\infty}^{\infty} \bar{F}\left(X^{\mu}\right) d^{4} X+O\left(\varepsilon^{2}\right) .
$$

Thus, if two-timing is appropriate and the $\psi^{A}$ are periodic in $\theta$, we may write

$$
I_{0}=\int_{-\infty}^{\infty} \bar{L}\left(X^{\mu}\right) d^{4} X+O\left(\varepsilon^{2}\right)
$$

Dougherty [5] has shown how this method may be extended to the case of a field consisting of a slowly-varying background and a weak rapidly-varying wave, so that $\psi^{A}$ has the form

$$
\psi^{A}=\psi_{0}^{A}\left(X^{\mu}\right)+e\left(a^{A}\left(X^{\mu}\right) e^{i \theta}+\bar{a}^{A}\left(X^{\mu}\right) e^{-i \theta}\right),
$$


where the bar denotes the complex conjugate. The relation between $e$ and $\varepsilon$ may affect the approximation scheme, as we shall later see it does in our case. This occurs in particular because the equation for $\psi_{0}^{A}$ is not (1.9), since $\psi_{0}^{A}$ is independent of $\theta$, but rather an equation involving a "back reaction" term dependent on $a^{A}$ and $\theta$. The effect of this term in general depends on the ratio of $e$ to $\varepsilon$. (Dougherty treated the particular case $e=\varepsilon$.) One may have to solve the equations for $\psi_{0}^{A}$ and the perturbation as simultaneous equations rather than solving successively for the different orders.

\section{Gravitational Waves and the Second Variation Lagrangian}

Einstein's field equations of general relativity are ${ }^{1}$

$$
G_{\mu \nu}:=R_{\mu \nu}-\frac{1}{2} R g_{\mu \nu}=T_{\mu \nu} .
$$

Since the full Riemann tensor can be written

$$
R_{\mu \nu \varrho \sigma}=C_{\mu \nu \varrho \sigma}+g_{\mu[\varrho} R_{\sigma] v}-g_{v[\varrho} R_{\sigma] \mu}+\frac{R}{6}\left(g_{\mu \varrho} g_{v \sigma}-g_{\mu \sigma} g_{v \varrho}\right)
$$

where the Weyl tensor $C_{\mu \nu \rho \sigma}$ is algebraically independent of $R_{\mu \nu}$, a nonzero Weyl tensor is considered to indicate the presence of fields (of Coulomb or radiative type) due to distant sources. The Weyl tensor is linked to the material energy-momentum $T_{\mu \nu}$ through the Bianchi identities

$$
R_{\mu \nu[\varrho \sigma ; \tau]}=0 .
$$

The non-linearity of these equations prevents us splitting the metric into parts due to different sources or into a background and a wave in general, i.e. we have no superposition principle. There is also no generally satisfactory definition of the energy of a gravitational field (Trautman and Pirani in [6]).

The usual way to discuss waves is to assume that as well as the real manifold $M$ there is a comparison (background) manifold $M_{0}$ and a map $\mu: M_{0} \rightarrow M$. One may introduce coordinates so that $p \in M_{0}$ and $\mu(p) \in M$ have the same coordinates, and it is assumed that then

$$
g_{\mu v}\left(x^{\kappa}\right)=\stackrel{\circ}{g}_{\mu v}\left(x^{\kappa}\right)+e g_{\mu \nu}^{\prime}\left(x^{\kappa}\right)+e^{2} g_{\mu v}^{\prime \prime}\left(x^{\kappa}\right)+\cdots
$$

${ }_{1}^{1}$ We adopt the conventions that the signature of spacetime is +2 , the units are chosen so that $8 \pi G=c=1$ and the Ricci identity reads

$$
b_{; \mu \tau}^{v}-b_{; \tau \mu}^{v}=-R_{\sigma \mu \tau}^{v} b^{\sigma} .
$$

$R_{\mu \nu}:=R_{\mu \sigma v}^{\sigma}, R:=R_{\mu}^{\mu}$. A comma separating indices denotes partial differentiation, a semi-colon covariant differentiation. Greek indices run from 1 to 4 . Square brackets surrounding indices denote skewing and round brackets symmetrisation. 
where $\stackrel{9}{g}, g^{\prime}, g^{\prime \prime} \ldots$ are of the same magnitude and $e \ll 1$. A coordinate change such that $p$ and $\mu(p)$ still have equal coordinates does not affect (2.4). If, however, one makes different coordinate changes in $M_{0}$ and $M$ and continues to identify points with equal coordinate values, this amounts to a change in the map $\mu$. A change of the form

$$
x^{\prime v}(\mu(p))=x^{\prime v}(p)+e \xi^{v}
$$

is called a "gauge transformation" because it alters the $g^{\prime}$ of (2.4) in a manner analogous to that of a gauge change of the potential in electrodynamics.

One can write

$$
G_{\mu \nu}=\stackrel{\circ}{G}_{\mu \nu}-\stackrel{w}{T}_{\mu \nu}
$$

where $\stackrel{\circ}{G}_{\mu \nu}$ is formed from $\stackrel{\circ}{g}_{\mu \nu}$, and call $\stackrel{\sim}{T}_{\mu \nu}$ the energy momentum tensor of the perturbation. This is not in general gauge-invariant, and indeed we should not expect it to be, since a gauge change of the form (2.5) alters the compared values of $G_{\mu \nu}$ and $\dot{G}_{\mu \nu}[7]$.

A case of particular interest is the high-frequency case where $\stackrel{\circ}{g}_{\mu \nu}$ varies slowly, with $n^{\text {th }}$ derivatives of order $\varepsilon^{n}$, and $g_{\mu \nu}^{\prime}$ varies rapidly with derivatives of order unity ${ }^{2}$ [8]. This property is only maintained after a gauge change if the additional terms contributed by the gauge transformation can also be split into high and low frequency components (cf. de Witt ${ }^{3}$ ). Within this restriction, the $\stackrel{w}{T}_{\mu \nu}$ is gauge-independent to lowest order in the approximation scheme ([8] and below).

The resulting equations have been studied under a variety of assumptions. Isaacson [8] takes $e=\varepsilon$ and equates coefficients of $\varepsilon$ in the equation $G_{\mu \nu}=0$ (cf. [2]). Madore [3] assumes $G_{\mu \nu}=0=\stackrel{\circ}{G}_{\mu \nu}$ and $\varepsilon^{2} \gg e$ and equates coefficients of $e$. In order to deduce that the coefficients of powers of $e$ or $\varepsilon$ vanish identically (rather than just being $O(e)$ or $O(\varepsilon)$ ) one could assume that there is a one-parameter family of solutions for varying $e$ and/or $\varepsilon[\mathrm{cf} .2,9,10,18]$. (For $M$ to be embedded in a one-parameter family of spaces $M_{e}$ such that as $e \rightarrow 0, M_{e} \rightarrow M_{0}$, one requires $\varepsilon \gg e$ when $e \rightarrow 0$.) The split into high and low frequencies (i.e. in effect the splitting of the derivatives of the terms into powers of $\varepsilon$ ) provides additional information about the equations of the approximation scheme.

It should be noted that (a) there is no generally accepted covariant rule for determining a suitable $\stackrel{\circ}{\mu \nu}_{\mu \nu}$ for (2.4) given $g_{\mu \nu}$, and (b) no coordinatefree characterisation of "high-frequency" has been offered in the literature (though the existence of sets of coordinates with specified properties is itself an invariant property of a space). The reason that despite these

${ }^{2}$ Since general relativity is invariant under an overall scale change (see e.g. [23]) only the relative magnitude of the terms is important.

${ }^{3}$ We thank Professor K. S. Thorne for informing us of this unpublished work and providing us with copies. 
objections our assumptions are interesting is that an experimenter who observed a rapidly varying field (through its effect on geodesic deviation) would naturally ascribe the variation to a high-frequency wave and attempt to construct a background by taking the averaged value of the curvature (introducing, in doing so, a preferred tetrad or coordinate frame). We also see that he would require a treatment that was gaugeinvariant in the restricted sense allowed by "high-frequency", since he would be uncertain of the exact identification between the real and the comparison space.

There are problems of interest in which one wishes to specify a class of space-times with metrics $\stackrel{\circ}{\mu \nu}_{\mu \nu}$ satisfying certain properties and consider all possible $g_{\mu \nu}$ obeying our assumptions. Thus point (a) above is negated. These instances usually also give an answer to point (b) since one can specify a length scale by considering the differential invariants of the space (e.g. the eigenvalues of the Ricci and Weyl tensors) and then compare the variation of $g_{\mu \nu}^{\prime}$ with this scale ${ }^{2}$. Thus there are problems where the objections just raised can be overcome.

For simplicity we shall restrict the discussion to the case of empty space, although the method is equally applicable whenever the matter content of the space obeys a Lagrangian description, for example if it were a perfect fluid or an electromagnetic field $[9,10]$. The Einstein equations are then the Euler equations of the integral

$$
I=\int R \sqrt{-g} d^{4} X .
$$

We substitute in (2.7) the expansion (2.4) and derive an expansion of $I$ in powers of $e$

where

$$
I=I_{0}+e I_{1}+\frac{e^{2}}{2} I_{2}+\cdots
$$

$$
\begin{aligned}
& I_{0}=\int \stackrel{\circ}{R} \sqrt{-\stackrel{\circ}{g}} d^{4} x, \\
& I_{1}=-\int \stackrel{\circ}{G}^{\mu v}\left(g_{\mu \nu}^{\prime}+e g_{\mu \nu}^{\prime \prime}\right) \sqrt{-\stackrel{\circ}{g}} d^{4} x, \\
& I_{2}=J_{1}+J_{2}, J_{2}=\int \mathscr{G}\left(\stackrel{\circ}{g}_{\mu v}, g_{\sigma \tau}^{\prime}\right) \sqrt{-\stackrel{\circ}{g}} d^{4} x, \\
& J_{1}=\int\left(2 \stackrel{\circ}{g}^{\sigma \alpha} \dot{\circ}^{\tau \beta}-\stackrel{\circ}{G}^{\sigma \tau} \dot{g}^{\alpha \beta}+\frac{\stackrel{\circ}{R}}{2}\left(\stackrel{g}{g}^{\sigma \alpha} \stackrel{\circ}{g}^{\tau \beta}-\frac{1}{2} \stackrel{g}{g}^{\sigma \tau} \stackrel{\circ}{g}^{\alpha \beta}\right)\right) k_{\alpha \beta} k_{\sigma \tau} \sqrt{-\stackrel{\circ}{g}} d^{4} x \\
& \mathscr{G}=\left(k^{\sigma \varrho ; \kappa} k_{\varrho \kappa ; \sigma}-\frac{1}{2} k^{\kappa \varrho ; \sigma} k_{\kappa \varrho ; \sigma}+\frac{1}{4} k^{, \sigma} k_{, \sigma}\right)
\end{aligned}
$$
and

$$
k_{\mu \nu}=g_{\mu v}^{\prime}+\frac{k}{2} \stackrel{\circ}{g}_{\mu \nu} ; k=-\stackrel{\circ}{\mu \nu}^{\mu v} g_{\mu \nu}^{\prime} .
$$

Here the semi-colon refers to covariant differentiation with respect to $\stackrel{\circ}{g}_{\mu \nu}$, and $\stackrel{\circ}{g}_{\mu \nu}$ and $\stackrel{g}{g}^{\mu \nu}$ are used to raise and lower indices. 
Let us consider the variational principle defined by the integral

$$
I_{0}+\frac{e^{2}}{2} J_{2}=\int\left\{\stackrel{\circ}{R}+\frac{e^{2}}{2} \mathscr{G}\left(\stackrel{\circ}{g}_{\mu \nu}, g_{\sigma \tau}^{\prime}\right)\right\} \sqrt{-\stackrel{\circ}{g}} d^{4} x .
$$

The Euler equations derived by varying (2.10) with respect to $g_{\mu v}^{\prime}$ are

$$
k_{(\mu ; \nu) \varrho}{ }^{e}-\frac{1}{2} k_{\mu \nu ;{ }^{\varrho}}-\frac{1}{2} \stackrel{\circ}{g}_{\mu \nu} k_{; \sigma \varrho}^{\sigma \varrho}=0 .
$$

(That the Euler equations derived by varying the $I_{2}$ in an expansion such as (2.8) with respect to the perturbation variables yields the same equations as perturbation of the governing equations derived from $I$ is a very general property of Lagrangian systems. $I_{2}$ is known as the second variation Lagrangian integral $[9,10]$.) The Euler equations derived from (2.10) by variation with respect to $\stackrel{g}{g}_{\mu \nu}$ are

$$
\stackrel{\circ}{G}^{\mu \nu}=e^{2} T^{\mu \nu}
$$

where $T^{\mu v}$ is obtained by varying $\stackrel{\circ}{g}_{\mu \nu}$ in $J_{2}$. The connection between this energy-momentum tensor and the "canonical" energy momentum is discussed in [10]. In this case the "canonical" energy-momentum is not symmetric, has indefinite sign of energy and does not possess the requisite gauge-invariance. Isaacson therefore rejected the Lagrangian approach [8], but it has been used by B. S. de Witt and by Kovacs ${ }^{3}$.

(2.12) shows that $I_{1}$ and $J_{1}$ are $O\left(e^{2}\right)$. The variation of $I_{1}$ with respect to $g_{\mu \nu}^{\prime}$ is $\stackrel{G}{\mu \nu}_{\mu \nu}$ which adds an $O(e)$ term to (2.11) while the variation of $I_{1}$ with respect to $\stackrel{\circ}{g}_{\mu v}$ adds a multiple of (2.11) to (2.12). Similarly the variation of $J_{1}$ does not alter (2.11) or (2.12) to highest order in $e$. Thus the variational principle given by (2.10) yields an approximation scheme which is selfconsistent up to terms of order $e^{2}$ in $G_{\mu v}$.

When the high-frequency approximation is introduced, this scheme is still correct provided $e \lesssim \varepsilon$. (If $e \gg \varepsilon$, the $\stackrel{\circ}{G}_{\mu \nu}$ term in (2.12) should be dropped, and $\stackrel{\circ}{\mu \nu}_{\mu \nu}$ would be determined from some higher-order terms in the scheme. Such a procedure would make little sense, for if $T^{\mu v}=0$ the perturbation must represent just a gauge transformation (see [8] and below).) It may be noted that if we were to solve $\stackrel{G}{\mu \nu}_{\mu \nu}=0$, use the solution in (2.11), compute $T^{\mu \nu}$ and then solve (2.12) for a modified background we would expect a correction of order $\frac{e^{2}}{\varepsilon^{2}}$ to $\stackrel{\circ}{\mu \nu}_{\mu \nu}$. Thus the concept of "back-reaction" only seems sensible if $e^{2} \ll \varepsilon^{2}$. Moreover the change of background would in general affect the solution of (2.11), in particular by altering the light-cones, along which we expect $k_{\mu v}$ to be propagated. 
It should be noted that we have so far placed no restriction of the usual kind (such as the harmonic condition) on the gauge. The latter is only required to give the high-frequency form, when that approximation scheme is applied.

\section{The Averaged Lagrangian for Gravitational Waves}

As we have already remarked, the "two-timing" method $[2,3]$ and the high-frequency approximation [8] (which is less restrictive in that the rapid variation of $g_{\mu \nu}^{\prime}$ need not be unidirectional) have been employed to discuss gravitational waves. As the remarks in Sections 1 and 2 show, these discussions could have been conducted by starting with the Lagrangian variational principle from (2.7). We seek here to employ the averaged Lagrangian technique, and we therefore assume a solution for $g_{\mu \nu}^{\prime}$ of the form (1.1) and periodic in $\theta$. In fact, since the Eq. (2.11) is linear in $k_{\mu \nu}$ and since the averaging will eliminate cross-products of terms in a Fourier series in multiples of $\theta$, we may restrict ourselves to the W.K.B. form

$$
g_{\mu \nu}^{\prime}=\alpha_{\mu \nu} e^{i \theta}+\bar{\alpha}_{\mu \nu} e^{-i \theta} .
$$

To help us keep track of the orders of the different terms, we shall use auxiliary variables $X^{\varrho}=\varepsilon X^{\varrho}$ and $\Theta=\varepsilon \theta$, so that the high-frequency approximation is expressed by $\varepsilon \ll 1$ and that derivatives of $\alpha_{\mu \nu}$ and $\Theta$ with respect to $X^{e}$ are of order unity, and we shall write

$$
b_{\mu: \varrho}:=\frac{\partial b_{\mu}}{\partial X^{\varrho}}-\bar{\Gamma}_{\mu \varrho}^{\kappa} b_{\kappa}=\varepsilon^{-1} b_{\mu ; \varrho}, \quad \text { etc. }
$$

where $\bar{\Gamma}_{\mu \varrho}^{\kappa}=\varepsilon^{-1} \Gamma^{\kappa}{ }_{\mu \varrho}$ is calculated by taking derivatives of $g_{\mu v}$ with respect to $X^{\varrho}$. This means we are evaluating $g_{\mu v}$ in the $x^{\varrho}$ coordinates and thereafter treating the components simply as functions ${ }^{4}$.

The averaged variational principle will give equations for $\alpha_{\mu \nu}$ and $\theta$ and (by varying $\stackrel{\circ}{\mu \nu}_{\mu \nu}$ ) an equation similar to (2.12) involving the "averaged stress-energy" of the waves. We stress that this has been arrived at by following the averaged Lagrangian method of Whitham [1] (i.e. averaging a scalar density) rather than by using the Brill-Hartle technique [11] for averaging tensors.

Substituting

$$
k_{\mu \nu}=a_{\mu \nu}\left(X^{\varrho}\right) e^{i \Theta\left(X^{\Theta}\right) / \varepsilon}+\text { c.c. }
$$

${ }^{4}$ We could equally well have used the functions $\tilde{g}_{\mu \nu}$ obtained by evaluation in the $X^{e}$ system, provided we made appropriate modifications to later equations. 
(where c.c. stands for complex conjugate) into the $J_{2}$ of (2.10) and carrying out the averaging, we derive the Lagrangian integral

$$
\begin{aligned}
\bar{I}=\int\{\stackrel{\circ}{R} & +\frac{e^{2}}{2}\left(\left[2 a_{\alpha}^{\sigma} \bar{a}^{\alpha \tau} l_{\sigma} l_{\tau}-\left(a^{\alpha \beta} \bar{a}_{\alpha \beta}-\frac{1}{2} a \bar{a}\right) l^{\sigma} l_{\sigma}\right]\right. \\
& +\varepsilon\left[2 i a_{\alpha \tau} l_{\sigma} \bar{a}^{\alpha \alpha: \tau}-i a_{\alpha \beta} l_{\sigma} \bar{a}^{\alpha \beta: \sigma}+\frac{i}{2} a l^{\sigma} \bar{a}_{, \sigma}+\text { c.c. }\right] \\
& \left.\left.+O\left(\varepsilon^{2}\right)\right)\right\} \sqrt{-\stackrel{g}{g}} d^{4} x
\end{aligned}
$$

where we use (1.2), and $\alpha_{\mu \nu}=a_{\mu \nu}-\frac{a}{2} \stackrel{\circ}{g}_{\mu \nu} ; a=a_{\mu \nu} \stackrel{\circ}{g}^{\mu \nu}$. The $O\left(e^{2} \varepsilon^{2}\right)$ terms in (3.3) arise both directly (as a term quadratic in $a_{\mu v: e}$ ) and from the correction terms to the average of the $O\left(e^{2}\right)$ terms (see (1.15)). The latter correction terms may be of still lower order [5] if suitable differentiability conditions are satisfied, and the $O\left(e^{2} \varepsilon^{2}\right)$ term would then be

$$
\int \frac{e^{2} \varepsilon^{2}}{2}\left[a_{\alpha \tau: \sigma} \bar{a}^{\alpha \sigma: \tau}-\frac{1}{2} a_{\alpha \beta: \sigma} \bar{a}^{\alpha \beta: \sigma}+\frac{1}{4} a_{, \sigma} \bar{a}^{\sigma}+\text { c.c. }\right] \sqrt{-\stackrel{g}{g}} d^{4} x .
$$

Since in deriving (2.10) we ignored $O\left(e^{3}\right)$ terms, it would not be valid to retain the $O\left(e^{2} \varepsilon\right)$ and $O\left(e^{2} \varepsilon^{2}\right)$ terms in (3.3) unless $\varepsilon \gg e$ or $\varepsilon^{2} \gg e$ respectively ${ }^{5}$. Varying the $O\left(e^{2}\right)$ terms with respect to $\theta$ and $a_{\alpha \beta}$ yields

and

$$
\frac{\partial}{\partial x^{\tau}}\left(\sqrt{-\grave{g}}\left(a_{\sigma}^{\alpha} \bar{a}_{\alpha}^{\tau}+a^{\tau \alpha} \bar{a}_{\alpha \sigma}+\frac{1}{2}\left(a \bar{a}-2 a^{\alpha \beta} \bar{a}_{\alpha \beta}\right) \delta_{\sigma}^{\tau}\right) l^{\sigma}\right)=0
$$

$$
2 a^{\tau(v} l_{\tau} \tau^{\mu)}-\left(a^{\mu v}-\frac{1}{2} a \dot{g}^{\mu v}\right) l^{\sigma} l_{\sigma}=0
$$

respectively. Contraction of (3.6) with $\bar{a}_{\mu \nu}$ shows that the extremal value of the $O\left(e^{2}\right)$ term is zero.

Now we can analyse (3.6), in a manner similar to Choquet-Bruhat [2], by taking two cases.

(a) if $l^{\sigma} l_{\sigma} \neq 0$ then let us introduce

$$
p^{\varrho \mu}=\stackrel{\circ}{g}^{\varrho \mu}-\frac{l^{\varrho} l^{\mu}}{l_{\sigma} l^{\sigma}} .
$$

${ }^{5}$ If the $O\left(e^{2} \varepsilon^{2}\right)$ and $O\left(e^{2} \varepsilon\right)$ terms are retained, and the Euler equations derived from each of them separately is used, the form (3.4) yields that $a_{\mu \nu}$ satisfies (2.11); this ensures that $a_{\mu \nu}$ satisfies the equation derived by varying $\theta$ in the $O\left(e^{2} \varepsilon\right)$ term, which is just (2.11) for $a_{\mu \nu}$ contracted with $\bar{a}^{\mu \nu}$; the equation found by varying the $O\left(e^{2} \varepsilon\right)$ term with respect to $a^{\mu \nu}$ is a propagation equation for $a_{\mu \nu}$ (which since we have not specified a gauge, involves a gauge condition); and the $O\left(e^{2}\right)$ term yields (3.5) and (3.6). Madore [3] has considered the equations found by taking the sum of these terms and varying $a_{\mu v}$, etc. (with a particular gauge), in order to discuss dispersion of gravitational waves. 
(3.6) can be written as

$$
a_{\varrho \sigma} p^{\varrho \mu} p^{\sigma v}=\frac{1}{2} a \stackrel{\circ}{g}^{\mu \nu}+\left(a_{\varrho \sigma} l^{\varrho} l^{\sigma}\right) \frac{l^{\mu} l^{\nu}}{\left(l^{\sigma} l_{\sigma}\right)^{2}}
$$

while its contraction gives

$$
2 a^{\tau v} l_{\tau} l_{v}+a\left(l^{\sigma} l_{\sigma}\right)=0 .
$$

Substituting back from (3.8) in (3.7) we obtain

which implies

$$
\left(a_{\varrho \sigma}-\frac{a}{2} \stackrel{\circ}{\varrho \sigma}_{\rho \sigma}\right) p^{\varrho \mu} p^{\sigma \nu}=0
$$

$$
\alpha_{\varrho \sigma}=2 l_{(\varrho} \lambda_{\sigma)},
$$

with $\lambda_{\sigma}$ of order unity, so that

$$
g_{\varrho \sigma}^{\prime}=2 l_{(\varrho} \lambda_{\sigma)} e^{i \Theta / \varepsilon}+\text { c.c. }
$$

This last is just the perturbation resulting from a gauge transformation

$$
x^{\prime \mu}=x^{\mu}+e\left[-i \lambda^{\mu} e^{i \Theta / \varepsilon}+\text { c.c. }\right] .
$$

(b) If $l_{\sigma} l^{\sigma}=0$ then

which implies

$$
\begin{gathered}
a^{\tau(\nu} l_{\tau} l^{\mu)}=0 \\
a_{\tau v} l^{\tau}=0 .
\end{gathered}
$$

Introducing a null tetrad $l_{\mu}, m_{\varrho}, t_{v}, \bar{t}_{\sigma}$ such that $l^{\mu} m_{\mu}=-1, t^{\sigma} \bar{t}_{\sigma}=1$ and all other scalar products are zero, we find

$$
\alpha_{\mu \nu}=l_{(\mu} \lambda_{v)}+\alpha t_{\mu} t_{v}+\beta \bar{t}_{\mu} \bar{t}_{v}
$$

where $\lambda_{v}, \alpha, \beta$ are arbitrary ${ }^{6}$ and the first term on the right again can be obtained by a gauge transformation of the form (3.11). If we write the Riemann tensor of the space as

$$
R_{\mu \sigma \tau \varrho}=\stackrel{\circ}{R}_{\mu \sigma \varrho}+e R_{\mu \sigma \tau \varrho}^{\prime}+e^{2} R_{\mu \sigma \tau \varrho}^{\prime \prime}+\cdots
$$

we find, on substituting (3.2) in the usual expression for $R_{\mu \sigma \tau}$, that

$$
R_{\mu \sigma \tau \varrho}^{\prime}=\frac{e}{2}\left(\alpha_{\mu \tau} l_{\sigma} l_{\varrho}+\alpha_{\sigma \varrho} l_{\mu} l_{\tau}-\alpha_{\sigma \tau} l_{\mu} l_{Q}-\alpha_{\mu \varrho} l_{\sigma} l_{\tau}\right) e^{i \Theta / \varepsilon}+\text { c.c. }
$$

and we note that a term of the form (3.9), which can be removed by a highfrequency gauge transformation, will not contribute to this, and that

${ }^{6}$ By using the freedom of a slowly varying initial phase of $\Theta$ and a slowly varying phase change $t_{\mu} \rightarrow t_{\mu} e^{i \gamma}$ we can make $\alpha$ and $\beta$ real and positive. The two parameters then yield the strengths of the two opposite circular polarisations of the wave. $|\alpha|=|\beta|$ yields plane polarised waves. 
it is of Petrov type $N$ with principal null direction $l_{\sigma}$ in the case $l_{\sigma} l^{\sigma}=0$ as we expect [8].

Varying (3.3) (ignoring the $O\left(e^{2} \varepsilon\right)$ and higher terms) with respect to $\stackrel{\circ}{g}_{\mu v}$ yields the Euler equation

$$
\begin{aligned}
\stackrel{\circ}{G}^{\mu v}= & e^{2}\left[-a^{\gamma(v} \bar{a}^{\mu) \tau} l_{\gamma} l_{\tau}+a^{\gamma(\mu} \bar{a}_{\gamma}^{v)}\left(l^{\sigma} l_{\sigma}\right)+\frac{1}{2}\left(a^{\alpha \beta} \bar{a}_{\alpha \beta}-\frac{1}{2} a \bar{a}\right) l^{\mu} l^{v}\right. \\
& -\left(\left[\bar{a}_{\gamma}^{\tau} a^{\gamma(\mu} l^{v)} l_{\tau}+\frac{1}{4} \bar{a}^{\mu v} a\left(l^{\sigma} l_{\sigma}\right)+\text { c.c. }\right]\right) \\
& +\frac{1}{4} \dot{g}^{\mu v}\left[2 a^{\sigma \alpha} \bar{a}_{\alpha}^{\tau} l_{\sigma} l_{\tau}-\left(a^{\alpha \beta} \bar{a}_{\alpha \beta}-\frac{1}{2} a \bar{a}\right) l^{\sigma} l_{\sigma}\right] .
\end{aligned}
$$

Again it is easily verified that terms of the form (3.9) do not contribute to (3.15) so that the stress-energy due to the perturbation (which is how we interpret the right side of (3.15)) is gauge-invariant. In the case $l_{\sigma} l^{\sigma}=0$ where this stress is non-zero, it reduces by (3.12) to

$$
\stackrel{\circ}{G}^{\mu \nu}=\frac{e^{2}}{2}\left(a^{\varrho \sigma} \bar{a}_{\varrho \sigma}-\frac{1}{2} a \bar{a}\right) l^{\mu} l^{\nu}=: \frac{e^{2}}{2} N l^{\mu} l^{\nu} .
$$

The remaining Euler Eq. (3.5), in the case of interest where $l^{\sigma} l_{\sigma}=0$, yields

$$
\left(\sqrt{-\dot{g}} N l^{\tau}\right)_{, \tau}=0
$$

which is just the contracted Bianchi identity for (3.16). It may be written

$$
(\log N)_{, \tau} l^{\tau}=-E
$$

where $E=l^{e} ; \varrho$ is the optical scalar of expansion. (3.17) thus is a conservation law for $N$ and shows [5], as we might expect since all our waves travel at the speed of light, that there is no dispersion. Substituting from (3.13) gives

$$
N:=a^{\varrho \sigma} a_{\varrho \sigma}-\frac{1}{2} a \bar{a}=\alpha \bar{\alpha}+\beta \bar{\beta}
$$

so that the wave energy-momentum has positive-definite energy, and is gauge-invariant. (It is easy to check gauge invariance under gauge changes with low-frequency components, or with high-frequency components of different propagation vectors.) Finally we should note that (3.17) shows that our approximations must break down as we approach any caustic of the congruence given by $l^{\sigma}$.

Our treatment covers, as we remarked earlier, any perturbation of the form

$$
g_{\mu v}^{\prime}=F\left(X^{\mu}, \theta\right)
$$

periodic in the rapid variable $\theta$. It can be extended to sums such as

$$
g_{\mu \nu}^{\prime}=\sum_{n}\left(\alpha_{\mu \nu}^{(n)} e^{i \boldsymbol{\theta}_{n} / \varepsilon}+\text { c.c. }\right)
$$

where $\stackrel{(n)}{\alpha}_{\mu \nu}, \Theta_{n}$ have derivatives of order unity with respect to $X^{\varrho}$, since, as Dougherty [5] has shown, the cross-product terms of differing $n$ 
vanish to lowest order in the averaging scheme, provided the propagation vectors $l_{(n)}^{\sigma}$ differ by terms of order unity. Thus our formalism extends, with this restriction on the propagation vectors, to sums of terms of the form (3.18). The averaged Lagrangian for (3.19) just contains a sum of terms similar to those appearing in (3.3) dependent on $a_{\mu v}$, the Euler equations for each $\stackrel{(n)}{\alpha}_{\mu \nu}$ and $\theta_{n}$ hold, and the energy-momentum tensors of the different waves simply add.

These results agree, wherever relevant, with the results of Isaacson [8].

Our discussion has ignored higher-order corrections. However, by returning to (2.8) and averaging, following Dougherty [5], one could take these into account. The next highest order terms are $O\left(e^{3}\right)$ terms from $I_{1}$ and $I_{3}$ plus the $O\left(e^{2} \varepsilon\right)$ terms in (3.3) that we ignored.

\section{Examples}

In this section we shall use the Robinson-Trautman metrics [14-16] as examples to demonstrate the concept of back-reaction, and spatiallyhomogeneous cosmological models (see e.g. [17]) as an example of the direct usefulness of the Lagrangian approach. Eq. (3.16) shows that we can add as the gravitational wave stress, a "null-fluid" with hypersurface orthogonal velocity vector. Such a stress-energy is conventionally considered as a directed flow of radiation [13] but it need not be. We can go further and add several such terms, as remarked in the previous section, and these may combine to simulate a fluid with timelike velocity.

Several examples of high-frequency gravitational waves have been given previously. The Vaidya metric has been considered in the selfconsistent scheme $(e=\varepsilon)$ by Isaacson [8] and Choquet-Bruhat [2], while Madore, taking $\varepsilon^{2} \gg e$ so that back-reaction can be ignored, has discussed plane waves in flat space and in the field of a plane [3] and in the Schwarzschild metric [12]. All these background spaces can be put into the Robinson-Trautman form ${ }^{7}$ [14-16]

where

$$
d s^{2}=\frac{\varrho^{2}}{p^{2}}\left(d \xi^{2}+d \eta^{2}\right)-2 d \varrho d \sigma-\left(K-\frac{2 m}{\varrho}-2 H \varrho\right) d \sigma^{2}
$$

$p=p(\xi, \eta, \sigma), m=m(\sigma), k=\Delta(\ln p) \quad$ with $\quad \Delta:=p^{2}\left(\frac{\partial^{2}}{\partial \xi^{2}}+\frac{\partial^{2}}{\partial \eta^{2}}\right)$ and

$$
H=\frac{\partial}{\partial \sigma}(\ln p) \text {. }
$$

The Ricci curvature is

$$
R_{\mu \nu}=\left(\frac{1}{2} \Delta K-2\left(\frac{\partial}{\partial \sigma}-3 H\right) m\right) \varrho^{-2} \sigma_{, \mu} \sigma_{, v} .
$$

${ }^{7}$ The coordinates here are the $X^{e}$ (cf. footnote 4). 
Since $\sigma_{, \mu}$ is a hypersurface-orthogonal null geodesic vector field in the metric (4.1) it is a suitable propagation direction for high-frequency gravitational waves, and the curvature (4.2) has the appropriate form (3.16).

Taking the coordinates as $\left(X^{1}, X^{2}, X^{3}, X^{4}\right)=(\xi, \eta, \varrho, \sigma)$ we find Eq. (3.12) yields

$$
a_{\tau 3}=0 \text {. }
$$

Removing the components of $a_{\mu \nu}$ corresponding to gauge transformations gives

$$
a_{\tau 4}=0 \text { and } a_{11}+a_{22}=0 \text {. }
$$

The Eq. (3.17) becomes

which implies that

$$
\left(\frac{\varrho^{2}}{p^{2}} N\right)_{, 3}=0
$$

$$
N=\frac{N_{0}(\xi, \eta, \sigma)}{\varrho^{2}}
$$

and the field equations are just

$$
\frac{1}{2} \Delta K-2\left(\frac{\partial}{\partial \sigma}-3 H\right) m=\frac{e^{2} N_{0}}{2 \varepsilon^{2}} .
$$

In this case we might think of a metric satisfying (4.5) with $N_{0}=0$ as the original background, and the solution of (4.5) as giving the modification due to the back reaction of the gravitational waves. It is consistent to do this only if the metrics and their differential invariants differ only slightly. Comparing (3.14) with the original Riemann tensor of the space we see this requires $e \ll \varepsilon^{2}$. This condition also ensures that the change in $\stackrel{\circ}{g}_{\mu v}$, which is of order $e^{2} / \varepsilon^{2}$, is smaller than the $O(e)$ perturbations we have discussed. If $e \ll \varepsilon^{2}$ does not hold, the "back reaction" cannot be considered as a small effect, and there will be no appropriate vacuum metric for comparison.

One case is the Vaidya metric, where $K=1, H=0$, so that

$$
\frac{\partial m}{\partial \sigma}=-\frac{e^{2} N_{0}}{4 \varepsilon^{2}} \text {. }
$$

By matching the invariant $K \varrho^{-2}[14]$ with the Schwarzschild metric we can view the difference of Vaidya and Schwarzschild as back-reaction when this concept makes sense (this identification matches the asymptotically flat infinities of the spaces).

Another interesting case is $p=1(K=H=0)$. Bonnor [16] has pointed out that this can be transformed (in the region $\varrho>0$ ) to

$$
d s^{2}=\tau^{4 / 3}\left(d \bar{\xi}^{2}+d \bar{\eta}^{2}\right)+\tau^{-2 / 3} d \zeta^{2}-d \tau^{2},
$$


the well known vacuum Kasner metric (a similar transformation in $\varrho<0$ yields the field of a plane [16]). If we take a modified metric and make the comparison by matching $m \varrho^{-3}$ (with, then, $m=1$ ) we find the varied metric, in the coordinates of (4.6) is

where

$$
\begin{aligned}
d s^{2}= & \frac{\tau^{4 / 3}}{p^{\prime 2}}\left(d \bar{\xi}^{2}+d \bar{\eta}^{2}\right)+\left(\tau^{-2 / 3}-K^{\prime}+H^{\prime} \tau^{2 / 3}\right) d \zeta^{2} \\
& -2\left(K^{\prime}-H \tau^{2 / 3}\right) \tau^{1 / 3} d \tau d \zeta-d \tau^{2}\left(1+K^{\prime} \tau^{2 / 3}-H^{\prime} \tau^{4 / 3}\right)
\end{aligned}
$$

and

$$
K^{\prime}=\frac{9}{4} \Delta^{\prime}\left(\ln p^{\prime}\right), H^{\prime}=3 \frac{\partial}{\partial \zeta}\left(\ln p^{\prime}\right), p=p^{\prime}\left(\bar{\xi}, \bar{\eta}, \zeta+\frac{3}{4} \tau^{4 / 3}\right)
$$

$$
3 \Delta^{\prime} K^{\prime}+4 H^{\prime}=\sqrt[6]{\frac{9}{16}} \frac{e^{2} N_{0}}{\varepsilon^{2}} .
$$

A limiting case of the metric (4.1) is the $p p$-wave form (Ehlers and Kundt [2])

$$
d s^{2}=d \xi^{2}+d \eta^{2}-2 d \varrho d \sigma-2 A d \sigma^{2}
$$

where $A=A(\xi, \eta, \sigma)$ and the Ricci curvature is

$$
R_{\mu \nu}=\frac{1}{2}\left(\nabla^{2} A\right) \sigma_{, \mu} \sigma_{, v}
$$

where $\nabla^{2}=\frac{\partial^{2}}{\partial \xi^{2}}+\frac{\partial^{2}}{\partial \eta^{2}}$. A gravitational wave with propagation vector $\sigma_{, \mu}$ and satisfying (4.3) is again allowed. (3.17) now gives

$$
N=N(\xi, \eta, \sigma)
$$

and the remaining field equation is

$$
\nabla^{2} A=\frac{e^{2} N}{\varepsilon^{2}} .
$$

Thus "back-reaction" preserves the $p p$-wave form and the source of Bonnor's metrics [13] could be gravitons rather than photons. From Bonnor's work we conclude that a graviton beam is stable and that two parallel graviton beams do not interact.

It should perhaps be noted that if $\stackrel{\circ}{\mu}_{\mu \nu}=\eta_{\mu \nu}$ is the metric of Minkowski space

$$
d s^{2}=d \xi^{2}+d \eta^{2}-2 d \varrho d \sigma
$$

and the gauge transformation

$$
x^{\prime \mu}=x^{\mu}+e H \eta^{v \mu} \sigma_{, v} \cos \sigma
$$

is made, then the metric (4.9) with

results.

$$
A=2 e H \cos \sigma
$$


Our other examples are spatially-homogeneous metrics. For the class $A$ metrics of [17], there is a Lagrangian formalism in which the metric is varied only within the class of spatially-homogeneous models [18]. To make use of this formalism we require the total stress-energy of the gravity waves to be spatially homogeneous. It is not clear whether and how one can make up such a stress-energy from a sum of terms of the form (3.16) which are not individually spatially-homogeneous ${ }^{8}$ so we restrict the discussion to cases where each element in any such sum is spatially-homogeneous. This condition implies that the vector $l_{\sigma}$ is invariant under the group of symmetries. In a basis each that the metric takes the form ${ }^{7}$

$$
d s^{2}=-\left(d x^{4}\right)^{2}+\gamma_{a b}\left(x^{4}\right) \sigma^{a} \sigma^{b}
$$

where the $\sigma^{a}(a=1,2,3)$ are 1 -forms in the coordinates $\left(x^{1}, x^{2}, x^{3}\right)$ invariant under the symmetry group and obeying

$$
d \sigma^{a}=C_{b c}^{a} \sigma^{b} \wedge \sigma^{c}
$$

and the $C_{b c}^{a}$ are the structure constants of the symmetry group, we must have the one-form $d \theta$, corresponding to $l_{\sigma}$, of the form

$$
d \theta=l_{0} d x^{4}+l_{1} \sigma^{1}+l_{2} \sigma^{2}+l_{3} \sigma^{3}
$$

where each $l_{\sigma}$ is a function of $x^{4}=t$ alone (by the homogeneity requirement). Thus we require

$$
d(d \theta)=0=d l_{1} \wedge \sigma^{1}+d l_{2} \wedge \sigma^{2}+d l_{3} \wedge \sigma^{3}+l_{a} C_{b c}^{a} \sigma^{b} \wedge \sigma^{c} .
$$

Choosing the $C_{b c}^{a}$ in the canonical way (diagonalising $\left.N^{a b}=\varepsilon^{c d(a} C^{b)}{ }_{c d}[17]\right)$ we find $l_{1}, l_{2}$ and $l_{3}$ must be constants and $N_{1} l_{1}=N_{2} l_{2}=N_{3} l_{3}=0$ (any null vector satisfying these conditions is geodesic). The possibilities are therefore; flow is any direction in type $\mathrm{I}$; in the 2 or 3 directions in type II $\left(N_{1} \neq 0, N_{2}=N_{3}=0\right)$; and in the 1-direction in type $\mathrm{VI}_{0}$ or $\mathrm{VII}_{0}$ $\left(N_{1}=0, N_{2} \neq 0 \neq N_{3}\right)$.

There are, therefore, a number of possibilities. For simplicity, let us restrict ourselves to flows exactly along the axes, with equal and opposite magnitudes propagating in the two opposite directions. These simulate a fluid (with anisotropic stress) moving perpendicular to the surfaces of homogeneity. If there is no net energy flow (i.e. $T^{4}{ }_{\alpha}=0$ ) the metrics can be diagonalised [19] so that

$$
d s^{2}=-d t^{2}+X^{2}(t) \sigma^{1} \sigma^{1}+Y^{2}(t) \sigma^{2} \sigma^{2}+Z^{2}(t) \sigma^{3} \sigma^{3} .
$$

8 That this may be possible is suggested by the asymmetric one-particle distribution functions for Robertson-Walker spaces, found by Treciokas and Ellis, which produce the required symmetry of the stress-tensor. 
Suppose the flow is along the 1-axis. Then the propagation vector for the positive direction is

$$
l_{\sigma}=\left(-\frac{1}{X}, 1,0,0\right)
$$

The Eq. (3.12) is satisfied, for this $l_{\sigma}$, if

$$
\frac{a_{v 4}}{X}-\frac{a_{v 1}}{X^{2}}=0,
$$

and the gauge transformation parts are removed if

$$
a_{v 1}=a_{v 4}=0 ; \quad \frac{a_{22}}{Y^{2}}+\frac{a_{33}}{Z^{2}}=0 .
$$

The conservation Eq. (3.17) yields

$$
(N Y Z)_{, 4}=0 \quad \text { implying } \quad N=\frac{M}{Y Z}, M \text { constant } .
$$

Combining the equal and oppositely-flowing wavetrains satisfying (4.14-15), we may write a Lagrangian integral,

$$
\int\left\{\frac{3}{2}\left(\dot{\beta}_{1}^{2}+\dot{\beta}_{2}^{2}\right)-6 \dot{\Omega}^{2}+R^{*}(\beta, \Omega)+\frac{e^{2} N}{2 \varepsilon^{2}}\left(l_{0}^{2}-l_{1}^{2} e^{2 \Omega-2 \beta_{1}}\right)\right\} e^{-3 \Omega} d t
$$

for the equations for $\Omega, \beta_{1}, \beta_{2}$; where (following Misner [20]),

$$
e^{-3 \Omega}=X Y Z, \quad X=e^{-\Omega+\beta_{1}}, \quad Y=e^{-\Omega+\frac{\beta_{1}}{2}+\frac{\sqrt{3} \beta_{2}}{2}},
$$

$R^{*}$ is the scalar curvature of the space sections, and we impose separately (since we are no longer varying $g_{44}$ ) the constraint that the corresponding Hamiltonian (which is time-independent) is zero, which is just the (44) field equation.

In Bianchi I one can add terms due to gravitational waves travelling along the other axes to simulate the stress-energy tensor of collisionless radiation which is initially isotropic [20], while is types $\mathrm{II}, \mathrm{VI}_{0}$ and $\mathrm{VII}_{0}$ one may add terms rather like the allowed forms for the stress-energy of magnetic fields [21-22]. In all cases the effect of the gravitational waves is to stabilise the universes against small shear anisotropies, just as the radiation [20] and magnetic fields [22] do. It is rather interesting that in types II, $\mathrm{VI}_{0}$ and $\mathrm{VII}_{0}$ the restricted terms one may add are precisely of the sort one wants in addition to the "geometric potential" derived from $R^{*}$. 


\title{
References
}

1. Whitham, G. B.: Studies in Applied Mathematics (ed. A. H. Taub), vol. 7. Mathematical Association of America Studies in Mathematics. Englewood Cliffs, N.J.: Prentice-Hall 1971.

2. Choquet-Bruhat, Y.: Commun. math. Phys. 12, 16 (1969).

3. Madore, J.: Commun. math. Phys. 27, 291 (1972).

4. Bretherton, F.P.: Proc. Roy. Soc. (London) A 302, 555 (1968).

5. Dougherty, J. P.: J. Plasma Phys. 4, 761 (1970).

6. Witten, L. (ed.): Gravitation; an introduction to current research, New York-London: John Wiley and Sons 1962.

7. Taub, A.H.: J. Math. Phys. 2, 787 (1961).

8. Isaacson, R. A.: Phys. Rev. 166, 1263 and 1272 (1968).

9. Taub, A.H.: Proceedings of the 1967 Colloque "Fluides et champ gravitationnel en relativité générale". Colloques internationaux du Centre National de la Recherche Scientifique (Paris) 170, 57 (1969).

10. Taub, A. H.: In: Relativistic Fluid Dynamics, ed. C.Cattaneo (Lectures at the Centro Internazionale Matematico Estivo, Bressanone, 1970) Edizioni Cremonese, Rome (1971).

11. Brill,D. R., Hartle, J. B.: Phys. Rev. 135, B 271 (1964).

12. Madore, J.: to appear (1973).

13. Bonnor, W. B.: Commun. math. Phys. 13, 163 (1969).

14. Robinson, I., Trautman, A.: Phys. Rev. Letters 4, 431 (1960); Proc. Roy. Soc. (London) A 265, 463 (1962).

15. Derry, L., Isaacson, R.A., Winicour, J.: Phys. Rev. 185, 1647 (1969).

16. Bonnor, W. B.: Physics Letters 31 A, 269 (1970).

17. Ellis, G.F.R., MacCallum, M. A. H.: Commun. math. Phys. 12, 108 (1969).

18. MacCallum, M.A.H., Taub, A.H.: Commun. math. Phys. 25, 173 (1972).

19. MacCallum, M.A.H., Stewart,J.M., Schmidt, B. G.: Commun. math. Phys. 17, 343 (1970).

20. Misner, C. W.: Astrophys. J. 151, 431 (1968).

21. Hughston, L. P., Jacobs, K.C.: Astrophys. J. 160, 147 (1970).

22. Jacobs, K.C., Hughston, L. P.: Paper in preparation (1972).

23. Penrose, R.: Structure of spacetime. Adams Prize Essay 1966, Princeton (1967).

\author{
A. H. Taub \\ Mathematics Department \\ University of California \\ Berkeley, California 94720, USA
}


